\title{
Model-Based Inference of Recombination Hotspots in a Highly Variable Oncogene
}

\author{
G. Greenspan, ${ }^{1}$ D. Geiger, ${ }^{1}$ F. Gotch, ${ }^{2}$ M. Bower, ${ }^{2}$ S. Patterson, ${ }^{2}$ M. Nelson, ${ }^{2}$ B. Gazzard, ${ }^{2}$ J. Stebbing $^{2}$ \\ ${ }^{1}$ Computer Science Department, Technion, Technion City, Haifa 32000, Israel \\ ${ }^{2}$ Department of Immunology, Division of Investigative Science, Faculty of Medicine, Imperial College of Science, \\ Technology and Medicine, The Chelsea and Westminster Hospital, London, United Kingdom
}

RE: J Mol Evol (2004) 58:239-251. A comma was mistakenly placed after the word "Highly" in the title of this article. The title appears correctly above. 